\title{
The Novel Modern Internet of Things System Structure Optimization Methodology based on Information Theory and Communication Signal Transmission Model
}

\author{
Huijun Yang \\ Department of Information Service, AnHui Institute of International Business, \\ Hefei AnHui, 231131, China
}

\begin{abstract}
In this paper, we research on the modern Internet of things system structure optimization methodology based on the information theory and the communication signal transmission model. We analyze the current IOT system from the following aspects. (1) Reliable delivery, that is, through some of the Internet and telecommunication network integration, it will send some information about the object accurately in real time; For the Internet of things, it is an integrated which is by a variety of network and the access and application technology of an integrated, at the same time it is also a can make the objects and people, nature and people as well as a platform for communication between objects and the objects. (2) The comprehensive perception which is to use some of the perception, capture and real-time measurement technology to information acquisition and access to an object with the sensing device can achieve data readout and data sent and no contact some automated to realize long-distance induction. (3) Intelligent processing, is one of the core content of the Internet of things, is to use some intelligent computing some analysis was carried out on large amount of information and data disposal, the Internet of things is able to intelligently control object by the computer network technology, micro processing technology, wireless communication technology and sensor technology product of development of fusion, therefore the Internet of things can be representative even instead of rational analysis. Through combining the information theory and the efficient signal transmission models, we optimize the current IOT architecture and the final experiment result indicates the feasibility of the proposed methodology.
\end{abstract}

Keywords: Internet of the Things; System Structure Optimization; Information Theory; Communication Signal Transmission Model; Mathematical Modelling

\section{Introduction}

The Internet of things is called after the computer, the Internet and the third wave of world information industry. Now many countries are spending a huge sum of money for the further research, the Internet of things is composed of the large number of information technology integration systems of new technology. It could be seen from the above our understanding of the Internet of things, the Internet of things is the extension and expansion of the Internet to the physical world and the Internet can be used as one of the important ways of Internet information transmission, the sensor network based on self-organization network, belongs to a kind of important perception technology in Internet of things. Internet of things with its basic properties, realizing any object, or anyone at the any time, any place will use any of the path networks and any connection of equipment. Therefore, the relevant properties including concentration, content of the Internet of things, collection, computing, communications and connectivity of the scene. These attributes is between people and the objects or the seamless connection between object and object [1]. The Internet of things is to point to by information sensing device, 
in accordance with the contract agreement, connect any item with the Internet, information exchange and the communication, in order to realize the intelligent identification, location, tracking and monitoring and management of a network [2-3].

A distinctive feature of the Internet of things is mainly manifested in the following aspects. Although the Internet of things to the radio frequency identification technology and all kinds of sensing device for the information collection, tracking, monitoring but it still cannot exist without the Internet. In the process of information transfer, either directly connected to the Internet, or using the wireless networks via their mobile phones, tablets and other electronic equipment is connected to a network [4-5]. Sensor has a pivotal position in the Internet of things. It can be better than the human senses to obtain more accurate and reliable information. Internet of things by means of the basic sensing technology, the track and the information in the test environment changes, through monitoring for the real-time data and information collection, update data, so as to realize information exchange and communication to provide intelligent control information. Networking besides can use sensors to obtain information, it itself is also more or less have the certain capacity to be able to obtain information and data for a certain processing and through the intelligent processor of objects of intelligent control [6]. In the figure one, we illustrate the general and basic structure of the Internet of things system.

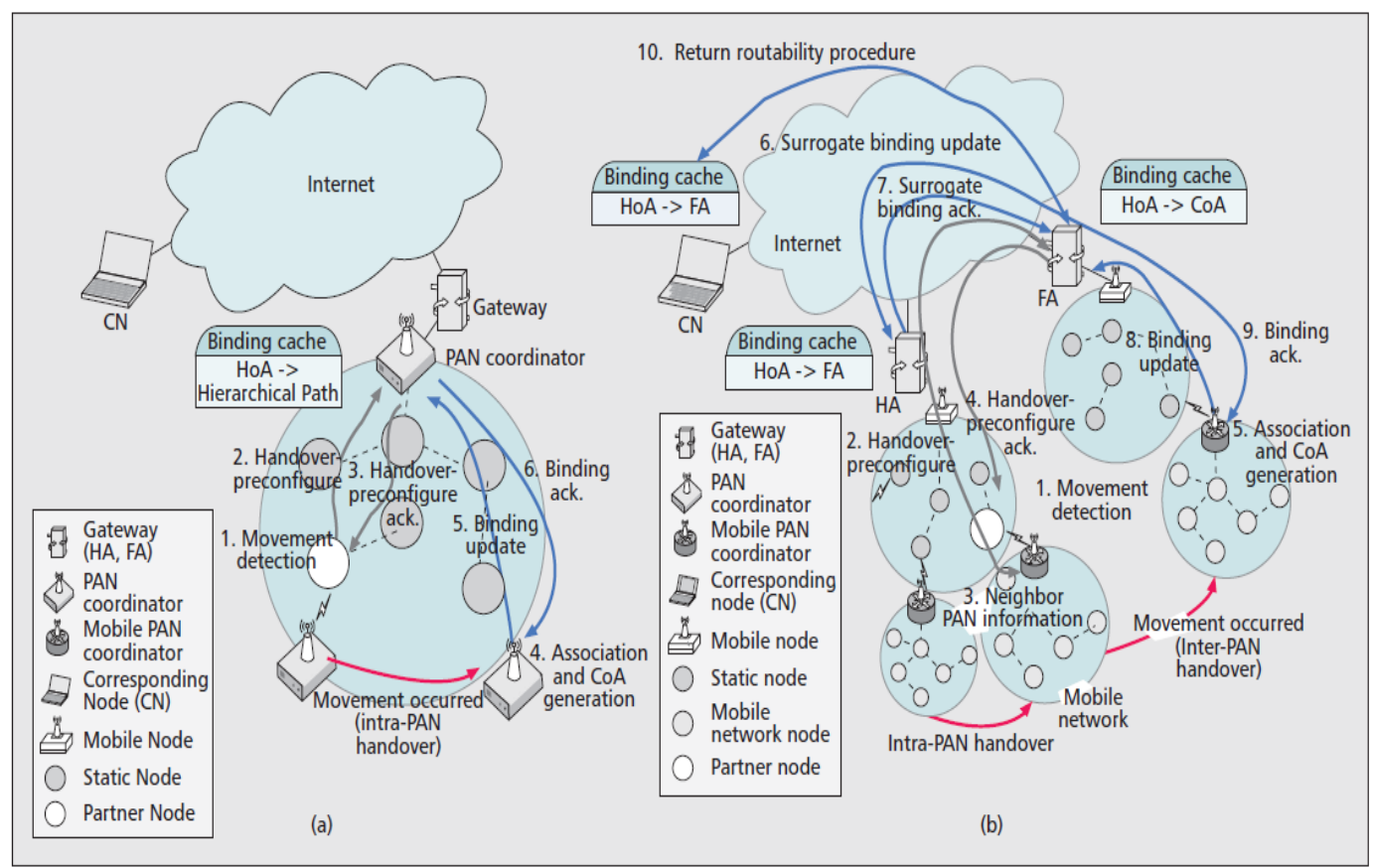

Figure 1. The General and Basic Structure of the Internet of Things System

As an emerging novel industry, the Internet of things in addition to covering the traditional industry in mobility convenience and environmental characteristics, their identification for all objects connection is actually reflected the idea of a unified and remote management, at the same time facing a turning point in the world economic development the traditional economic propulsion engines under the background of weak, Internet of things is a kind of tide at the head of the economic development of a new era. The emergence of the Internet of things will not only bring fundamental changes to the production activities of human society, at the same time, it will fundamentally change People's daily life, from experience of direct perception, people can more convenient to learn the living environment around the people and understand everything would like to know status and within scope allowed for its detection and remote control of relevant 
things. Reflect on and in the social effect which can realize life application of environmental protection, intelligent, accurate and safe. The Internet of things is finalized by sensor network, RF tags, bar code and the QR code reading device equipment and Internet, etc. The current technical development is not balanced, RF label, bar code and the QR code technology has been mature and sensor network technology has great development space [7].

To sum up, the essential techniques adopted by the Internet of things system could be listed as the follows. (1) Intelligent sensing technology. Data acquisition is the Internet of things "of things, figure interaction". Collection devices typically have controller. Due to low cost limit, so generally uses the general embedded systems. Specification requirements of the Internet of the terminal device must be a smart and so the information collection equipment generally has operating system. (2) Intelligent interactive and collaborative awareness. In the Internet of things intelligent interaction mainly reflects on the key technology in scene perception and physical signals and biochemical signals by making decisions about the different event and adjusts their own behavior, therefore it has become an indispensable part of IOT application system. At same time, situational awareness can make some data in Internet of things in low energy consumption way processing on local resource constraints of sensor nodes thus make the whole network energy consumption and minimize bandwidth. (3) Information processing and information fusion. Because the general Internet of things has obvious requirements and characteristics of "intelligence", and intelligent information processing is the guarantee of the properties of the common key technology, so the related key technologies of intelligent information processing and the research foundation for the development of the Internet of things has important role. Information fusion is an important stage of intelligent information processing and fusion is the multi-level, multi-faceted which will come from multiple data sources in the sensor network data processing process. (4) Security. Because of the Internet of things terminal sensing network of private property, so safety is a must face the problem. In the IOT sensor nodes usually needs to be deployed in unattended, uncontrolled environment, aside from general wireless network of information disclosure, tampering, replay attack, denial of service, and other threats also face sensor nodes are easy to be an attacker to obtain, through physical means to get all information stored in node, thus threat of invasion, control network. From the angle of safety technology, related technologies, including ensuring the safety of the user identity authentication technology as the core, to ensure the safe transport of key establishment and distribution mechanism, and ensure safety of data itself data security technology such as data encryption and the data security protocol [8]. The overall summary of the adopted techniques and tools are listed and shown in the following figure two. 


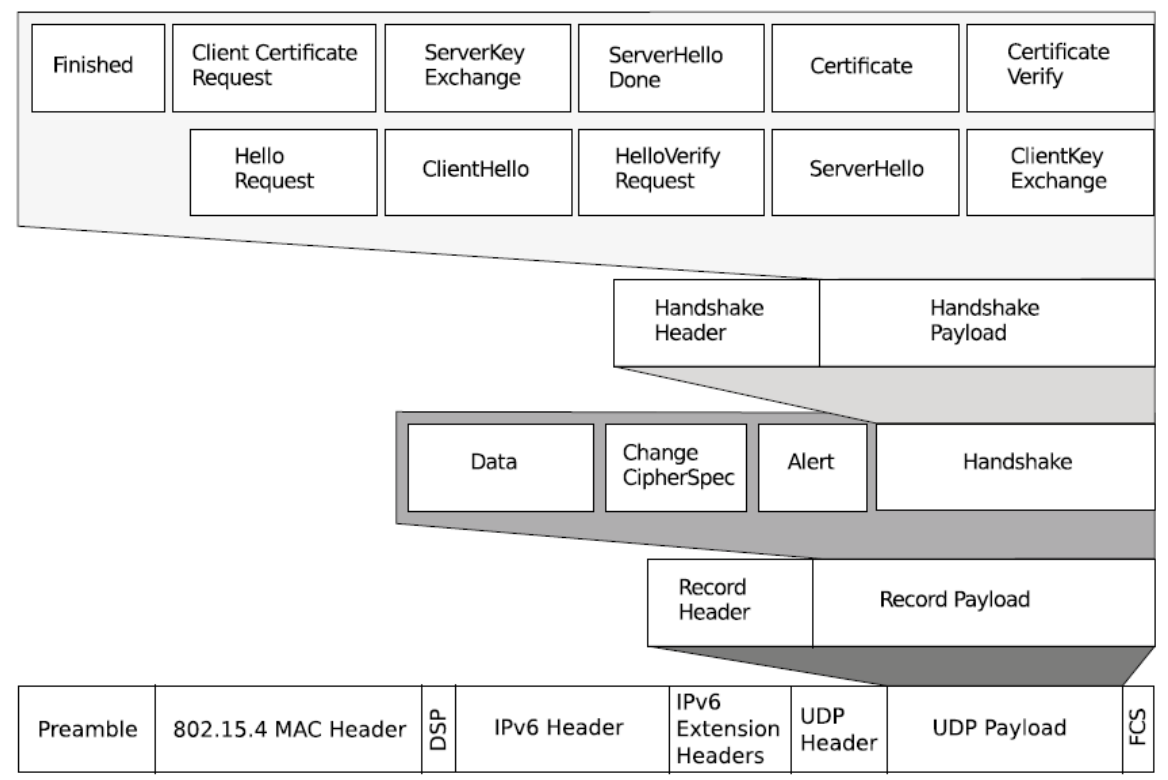

Figure 2. The Essential Techniques Adopted by the Internet of Things

To deal with the mentioned challenges and drawback, we research on the modern Internet of things system structure optimization methodology based on the information theory and the communication signal transmission model in this paper. This research paper is organized as the follows. In the first section, we introduce research background and the current research model. In the section two, we theoretically analyze the primary features and the structure of the Internet of things system with general description. In the sections three and four, we introduce the information theory and communication signal transmission model into the research. In the section five, we illustrate the optimized and the modified structure of the Internet of the things system with numeral simulation on the security, effectiveness and robustness. As final conclusion, we summary our work and give prospect for future research.

\section{Primary Features and the Structure of the Internet of Things System}

The working principle of the Internet of things is to rely on with intelligent sensor devices or other intelligent terminal, the communication network technology is used to analyze the information on the Internet a cloud computing model integrated computing. Information on the information to make use of the Internet of things computing or storage that not only can get the effect of stable and reliable, also can realize the combination of a variety of ways the personalized. According to the basic analysis and understanding, the Internet system structure of the content are discussed from several angles [9-11].

(1) The network layer. In the Internet, the network layer plays the role of communication and coordination, and relies on the ways and means is often applied to the Internet or call it wan communications systems. Using the Internet, the information gathered by the perception layer can flexible application in the network layer, a network extending in all directions found in all corners of the earth and this information will also be through the network layer reach the every need them, really realize the extreme distance and large range of communication. Service layer mainly provides a common interface and agent service, data general interface layer is responsible for parsing the data call instruction, shielding different database and data formats, and favorable for various database distributed deployment. Application of common interface can contact the upper consumers and its main purpose is for the different types of users are using communication protocol parsing, realize compatibility various kinds of communication 
protocol. With human society and the continuous development of Internet technology, is the future trend of the development of information age and the human life gradually replaced by information, therefore, in the future life, the amount of data in the Internet of things will become very large and complex.

(2) The application layer. In the application layer, the role of the Internet to collect and share the information collected. The general status of the application layer in the Internet of things is equivalent to the control hub, for the content of the whole system to make decisions and to provide information. A service is the purpose of the Internet of things work, through the application layer people can get their needs and sharing information. On some data and information collection work is completed, the information will be in the core application layer system complete summary, and through specialized study and, according to the measuring system and organization to identify reasonable information available, the useful value of comprehensive utilization of information to the human, to ensure maximum use of information and application in practice, it is mainly used for medical transport, logistics, power and so on intelligent information industries.

(3) The perception layer. The level of awareness in the Internet of things play a role of the gathering data, the data information contains the content of the audio and video information, in addition to this identity information, physical information, and so on. In the process of collecting, RFID and sensor technology is the main application methods of network data acquisition. With the perception layer, items will rely on the information communication to express the own value and human beings. Sensory data and control commands the two-way communication of the information, the general said is the network layer mainly on information obtained from perception layer real-time transmission, storage and processing.

Coming from the upper network transmission control information will also be using technologies such as smart sensors for storage and then use of language of the Internet to complete, in some ways, this way is just like human mouth, the effect of information on this kind of method to express their own opinions. In figure three, we demonstrate the primary features of the Internet of things systems.
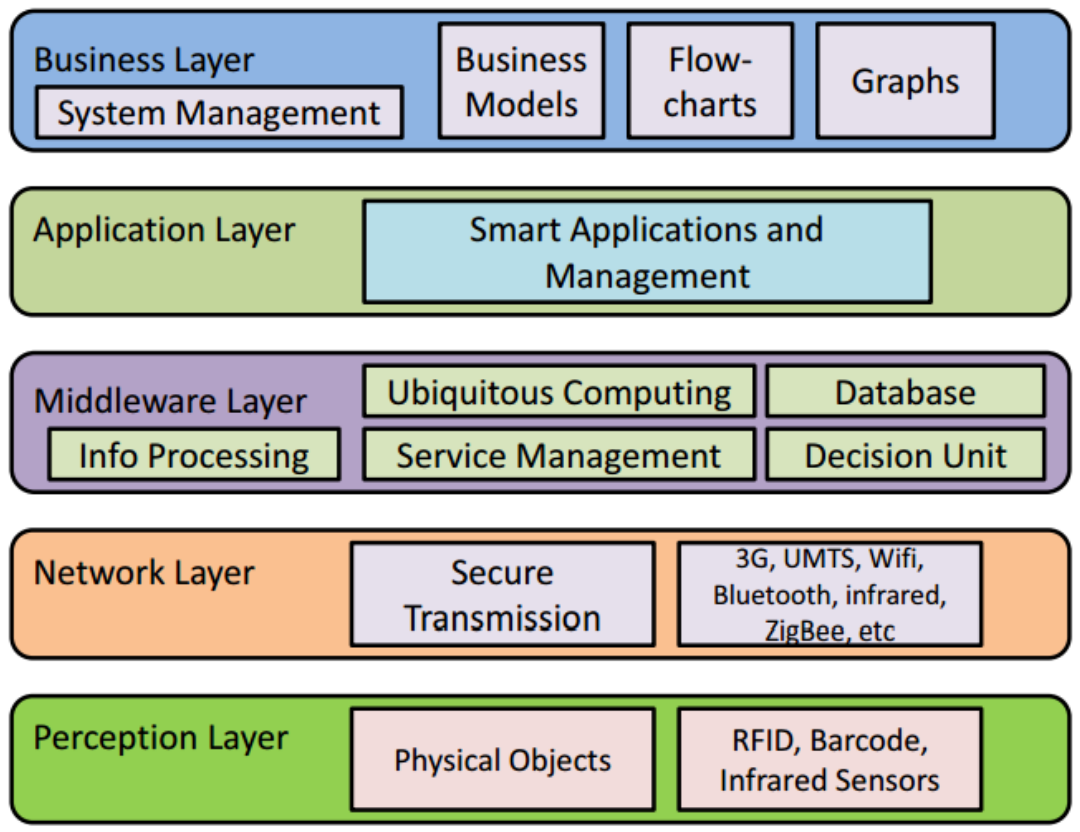

Figure 3. The Primary Features and Layers of the Internet of Things
Systems 
The Internet of things can be divided into public Internet of things and special things which can public Internet of things refers to connect all of the items, covering a social administrative area and the public Internet connection, as real social public information infrastructure of Internet of things. Dedicated Internet of things refers to the connection of certain items, covering a particular area, as a special use of the Internet of things within the agency. From the point of view of the physical world and the online world fusion, technical involves technology can be divided into the objects of information technology including the typical technology is the information materials. Information sensing and items behavior control technology, which is characteristic of the Internet of things application network physical system technology. The general of the information transmission, processing and decision technology, this is the Internet does not have the existing technology is geared to the needs of the physical world processing technology, virtual network can be referred to as physical network technology. Network architecture is mainly to research the composition of the network components as well as the core relationship between these components [12]. According to the researchers concerned about the angle of network system is different which can be divided into the different types of network architecture. From the standpoint of the function of the network, for example, we can get the function of network layered architecture from the general perspective of the network management can get the network management architecture.

\section{The Information Theory for Optimization}

The essence of the Shannon information theory is the abstract of the core content of the communication of information, the information given in the form of mathematics, the information processing in the process of communication into a strict mathematical operation. If a source of actual information entropy coding space occupied less than its, so, can the source parameters are a kind of coding compression mathematical transformation, make new coding occupied space can be compressed to near or even equal to the original source of the information entropy. In this way, we could complete the communication with fewer channel capacity without loss of the information. If there is interference in the channel, increase the amount of supervision code corresponding to the error correction and guarantee the lossless transmission of information. The final at the receiving end, still need to do the mathematical inverse transform decoding to fully recover original signal. This is Shannon letter the basic mathematical principle of source coding and channel coding. As for Internet of things based system and the sensor networks receiving signal is expressed as the follows [13].

$$
X(t)=A S(t), \quad M \geq N
$$

Physical characteristic parameters are actually is objective existence, they are in the certain time point values should also be the only one. But before accurate cognition, the cognition of its values often cannot be determined generally can be used on the set of values of probability distribution function to describe them which is shown below.

$$
W=E\left\{X g\left(W^{T} X\right)\right\}-E\left\{g^{\prime}\left(W^{T} X\right)\right\} W^{*}
$$

Regularity information usually use to describe the conceptual statements; you can also use mathematical equation to describe. These statements can be deterministic, can also be a probable. Whether it is a statement or formula itself with a symbol to prepare, and symbols will inevitably exist in the brain in the form of the corresponding data coding. Therefore, every can use symbols to reflect the rules of programming and itself is still a data file this data file is the application of the law which is shown below.

$$
\Delta W=\xi\left(W^{-T}-\hbar(Y) X^{T}\right) W^{T} W
$$


If at a signal in space domain with fixed constraint each other signal was detected in data collection, and the probability of the data sets appear far outweigh the possible probability, under the condition of the disorder in the signal space, cognitive is observed in a "like". The "like" can be static images, can also be a dynamic image for voice signal, it may be a thunder or the human voice and continuous movement, sensory signals, such as temperature, contact pressure will produce corresponding to "like". Under normal circumstances implementation of mixed signal separation is the most direct method for mixed signal mixed matrix inverse solution of mixed, but this method needs to know that mixing matrix. When the signal is a set of cannot directly to establish the mathematical model of mixed signal to the collected the signals, or lack of prior knowledge but know signal source is independent of each other a few mixed signal, fanaticism, separation is first choice for solving signal separation. To overcome the drawbacks, we use the following steps to help optimize the basic steps.

$$
\begin{gathered}
G[u(t)]=E\left[\exp \left\{\int \log u(t) d N_{t}\right\}\right] \\
G^{(M)}[u(t)]=E\left[\exp \left\{\int \log u^{\prime}(t) d N_{t}\right\}\right]
\end{gathered}
$$

Actual cognition, the brain is not to do after all the signal data obtained processing, but the signal is input, whenever there is a perception at the perceptual level as soon as quickly and timely analysis of the calculation, the relevant information and content information extracted, and according to the need to command one's own actions and at the same time, it will store the important information memory. Label provides sensory properties of data collection, the direction of the location and time of perception, perception and other information in the data collection, the serial number provided to homogeneous signal discrete space and the sampling sequence of discrete time and other information and the data value was in a certain sense of precision comparison signal of quantitative values. The rest of the basic stray chaotic signal and the useless information is basic, to save the storage space of the brain. Then, the brain again in thinking level, knowledge reasoning in inductive and deductive methods, establish and enrich theory knowledge, use the theoretical knowledge to guide their practice and from the practice of inspection to the correct and improve the existing knowledge system [14]. In the following figure, we show the information theory assisted network balancing mechanism.

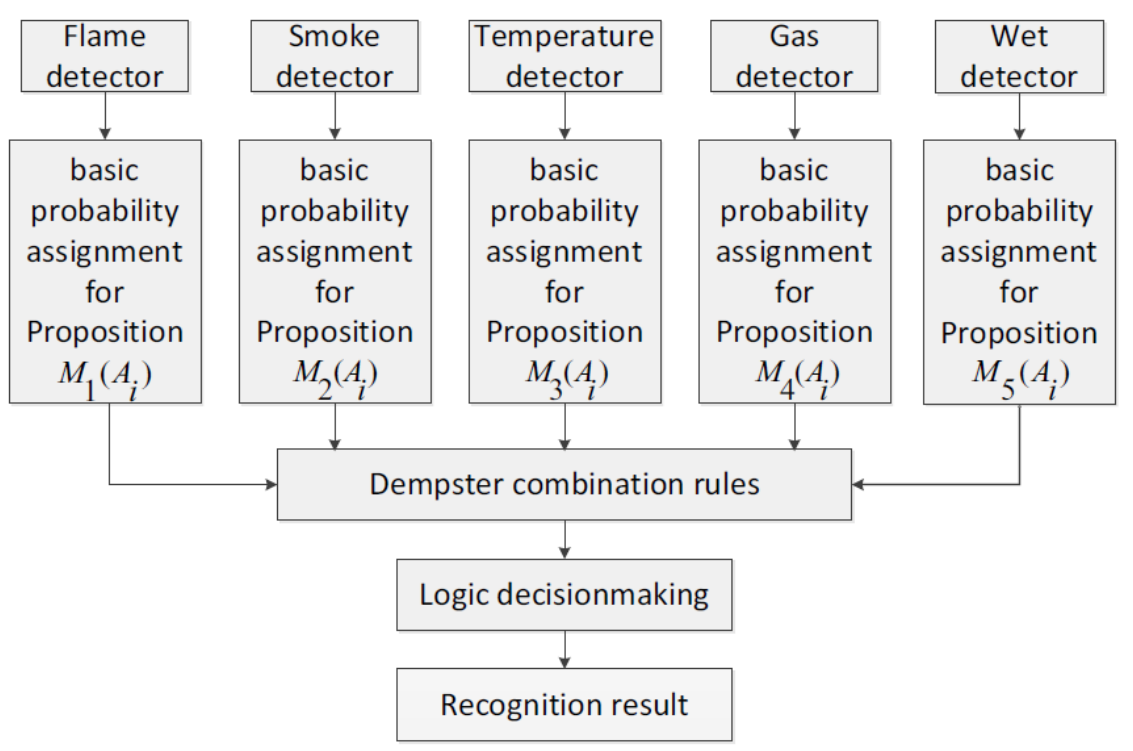

Figure 4. The Information Theory Assisted Network Balancing Mechanism 


\section{The Communication Signal Transmission Model}

The machines and machine communication refers to the general use of automatic control and network communication technology, in absence of government intervention to achieve autonomous data communication and information interaction between machine and machine series of the floorboard of the technology or technology combination. It is for the variety of terminal equipment between systems, network connection and the real-time communication between remote entities which provides effective way to transfer data. At present, according to the Internet security transmission problem, many scholars have carried on the thorough research and successively put forward the core series of typical methods. In the process of transmission, remote objects information server in response to the path of each node in the order from the back to before the nesting of item information with the public key encryption. The encrypted data each layer through a routing node decrypted, it was only local information server items back into clear each routing node in the process of transmission can verify the integrity of the received data and the authenticity of the forwarding path. In the following two equations, we define the basic transmission pattern of the signals [15].

$$
\begin{gathered}
y(t)=\text { Hjam } \rightarrow \text { recj }(t)+H \text { self } x(t) \\
y(t)=\text { Hjam } \rightarrow l j(t)+H r e c \rightarrow l x(t)
\end{gathered}
$$

To help finalize the whole system, we use following parts to assist. (1) The search process. Terminal after enters the region communication network, the first network access nodes information around search, access to the node of the search process is the terminal scanning process of channel access nodes of network information. In the process, terminal on a channel request message, and then wait for the access point, and then turn to the next channel. Standard regulation, after receipt of a request message of terminal access nodes, must reply bag to carry their own information to the terminal, terminal successfully after receiving the reply access nodes, can know the existence of the access node and obtain relevant information access points. (2) The associated process. After certification, you need to access nodes weight associated with the connection on, and then through the access node is connected the Internet. In the process of heavy association, terminal to send heavy association request frame to access nodes, the frame contains some information terminals, such as terminal supported by the data bit rate and the terminal will connect access nodes in the network SSID. After receives the request, access node processing request and build a connection ID number for the terminal part and allocates storage space to store information. Then send terminal access node weight associated response to terminal success or failure, and contains information about access nodes to establish a link between heavy. If the association is successful, the terminal can use this access nodes connected to the Internet and communicate with other is connected to the terminal. (3) The certification process. On a connection terminal a new access points, need to access the node authentication. After entered the stage of certification, access to the node sends a first to verify the basic contents of the fragment to the terminal and the terminal after this content will parse results back to access nodes, to judge whether the content of the terminal parsed correctly, if the right through the authentication, otherwise failure. The topology of the process is shown in the figure 5 . 

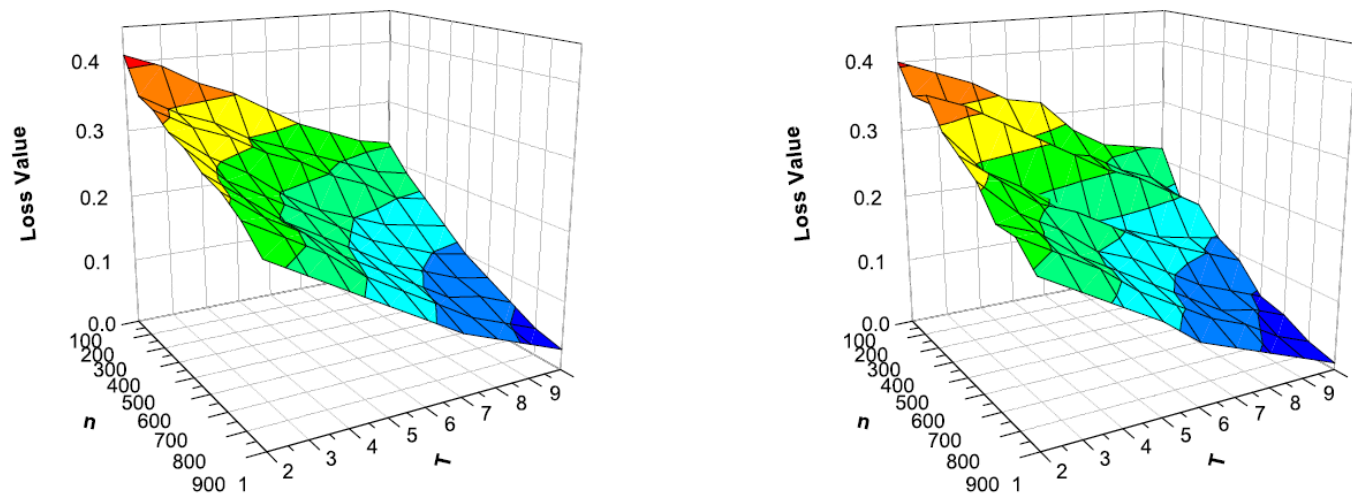

Figure 5. The Topology of the Communication System for Analysis

For any shape parameters of the first kind of confluence hypergeometric function is still no uniform analytic expression, therefore the following expression solve the issue.

$$
\begin{gathered}
A \approx G x \quad \text { where } \min _{x} \sum_{i}\|x\|_{0}:\|B-G x\|_{F}^{2} \leq \varepsilon \\
O_{k}=\bar{w} f_{k}\left(d_{0}^{k}+d_{1}^{k} x_{1}+d_{2}^{k} x_{2}+\ldots+d_{m}^{k} x_{m}\right)
\end{gathered}
$$

Observation field a fixed main function is to transmit the lightning current to the earth. Under normal circumstances, the network is designed for closed loop around the periphery of the fence, grounding installation outside the fence to ensure the safe distance between the data cable and ground wire. The observation on all of the devices must be connected to the ground network to realize strong [16]. The measurement standard is shown as the follows.

$$
C C=\left|\frac{\left(\sum_{x, y} B^{\prime}(x, y) A(x, y)-M N \mu_{B^{\prime}} \mu_{A}\right)}{\left(\sum_{x, y} B^{\prime 2}\left(x, y-M N \mu_{B^{\prime}}^{2}\right)\right) \times\left(\sum_{x, y} \mathrm{~A}^{\prime 2}(x, y)-M N \mu_{A}^{2}\right)}\right|
$$

A processor is used to receive perception layer network data and network to send data to the perception layer, two processors parallel work. Using dual port RAM as data buffer zone, it has two sets of completely symmetrical address line and data line and control line, allowing two processors to access memories at the same time, the communication on the basis of resource sharing. The fabric of the Internet of things including network system, the gateway and the Internet and wireless sensor network for data communication between networks and among them, the gateway is an important component of the Internet of things, as the core of the management, control and communicates with the external network. It is connected to the internal network and external network physical interface, realize the internal and external data exchange function and the question of communication network is also responsible for the management of equipment and control which is denoted as the follows [17].

$$
\left\{\begin{array}{c}
\nabla w^{(l)}=\frac{1}{m} \cdot \sigma^{(l+1)} \cdot\left(\sigma^{(l)}\right)^{T}+\lambda w^{(l)} \\
\nabla b^{(l)}=\frac{1}{m} \sum_{i=1}^{m} \sigma^{(l+1)}
\end{array}\right.
$$

Use the label only needs to realize the pseudo random number generator to construct a simple RFID authentication protocol SFP, and proves the SFP agreement under the standard model with the forward privacy security. Simulation experiment results show that compared with the existing RFID protocols, SFP protocol not only computation and 
communication with high efficiency, and at the same time guarantee the forward security and privacy label production costs low, implement the RFID tag cost low and the combination of high privacy. However, the above method still has many disadvantages, such as tags embedded in any of items, users in the absence of notice the label by reader scanning, through the item's location can track the user's whereabouts, thereby causing damage to the user privacy, and as the RFID system to read fast, to quickly scan way can track the change trend of enterprise sales, to steal trade secrets. Transport layer is to solve the data gained by the perception layer in a certain range, is usually a long-distance data transmission problem, mainly complete access and transmission function. The sensory information after local LAN network convergence by communication gateway and wide area network interconnection and realize the long-distance data transmission. Our method will enhance the transmission robustness.

\section{The Experimental Analysis}

In this section, we firstly propose our designed novel structure for the IOT system with the flowchart description and demonstration. Later, to prove the effectiveness and the robustness of the proposed system, in the second sub-section, we numerically simulate proposed method from the perspectives of structural efficiency.

\subsection{The Optimized Structure of the IOT System}

In the following figure 6 , we illustrate the general structure of the proposed system. For the Internet of things, though, its operation is based on the Internet for the real-time running and operation, but the Internet of things in the process of running for the stability of the control system is very demanding. The operation of the Internet of things should be starting from the implementation method, and improve application effects. To make up with the requirement, our design strictly follows the standards. (1) In the process of the design of Internet of things, also should guarantee the safety of principle, in the architecture of Internet of things, have to be regular operating system, the network maintenance dredging of the remote control signal through a network channel, for the large scale network attack provide effective defense. (2) To follow the principle of diversity in the structure of the Internet of things, in the process of architecture design, should according to different label node to service type of corresponding design, in order to meet the expected control requirements as the prerequisite, innovative design and analysis. (3) In the Internet of things in the process of architecture design, follow the principle of interconnectedness, establishment of objects with the Internet communication channel, keep IOT architecture exist many differences. Only obey the mentioned standard, the optimal structure for the robust IOT system will be achieved. 


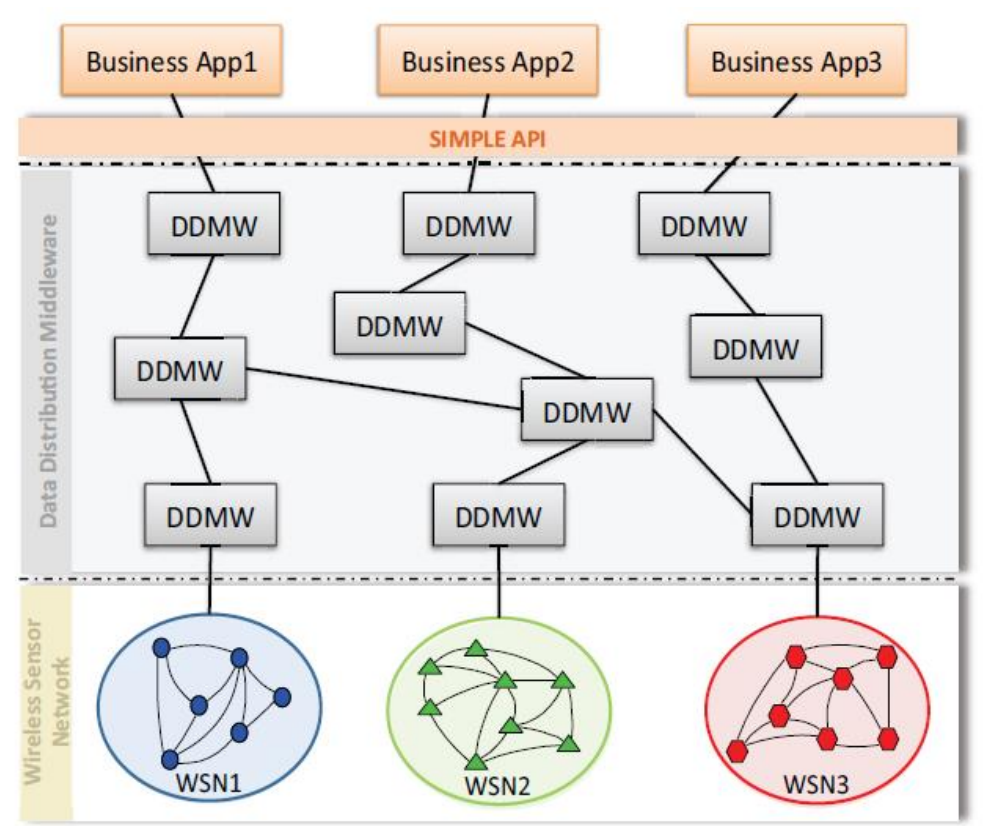

Figure 6. The Structure of the Proposed Optimal IOT Architecture

\subsection{Numerical Simulation and Experiment}

In this section, we numerically simulate the mentioned methodology and figure 7 shows the result. We could conclude from the simulation result that the optimized architecture will enhance the overall performance of the traditional IOT systems.

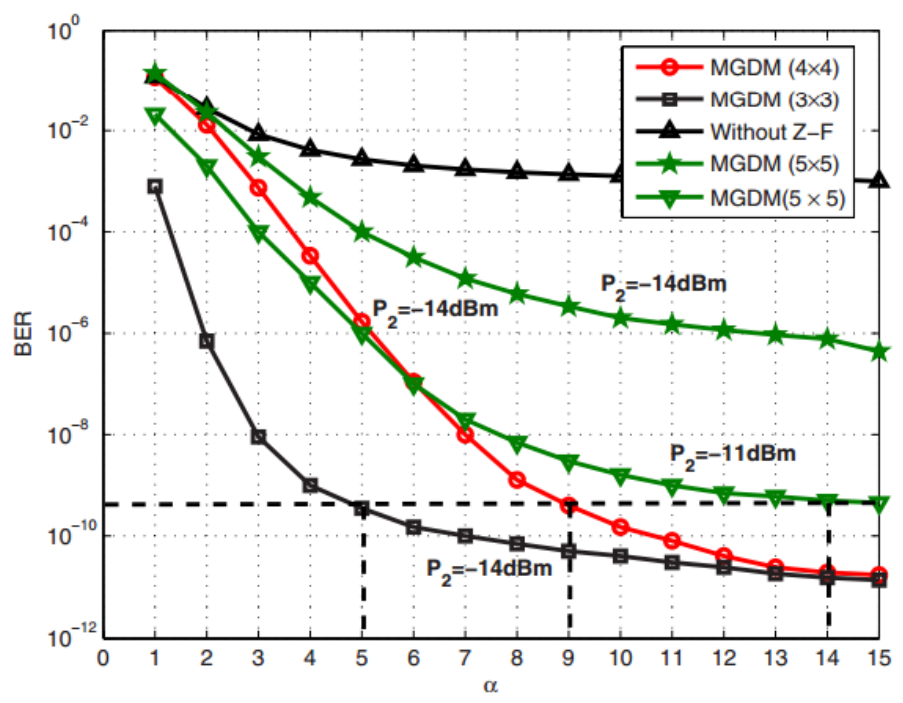



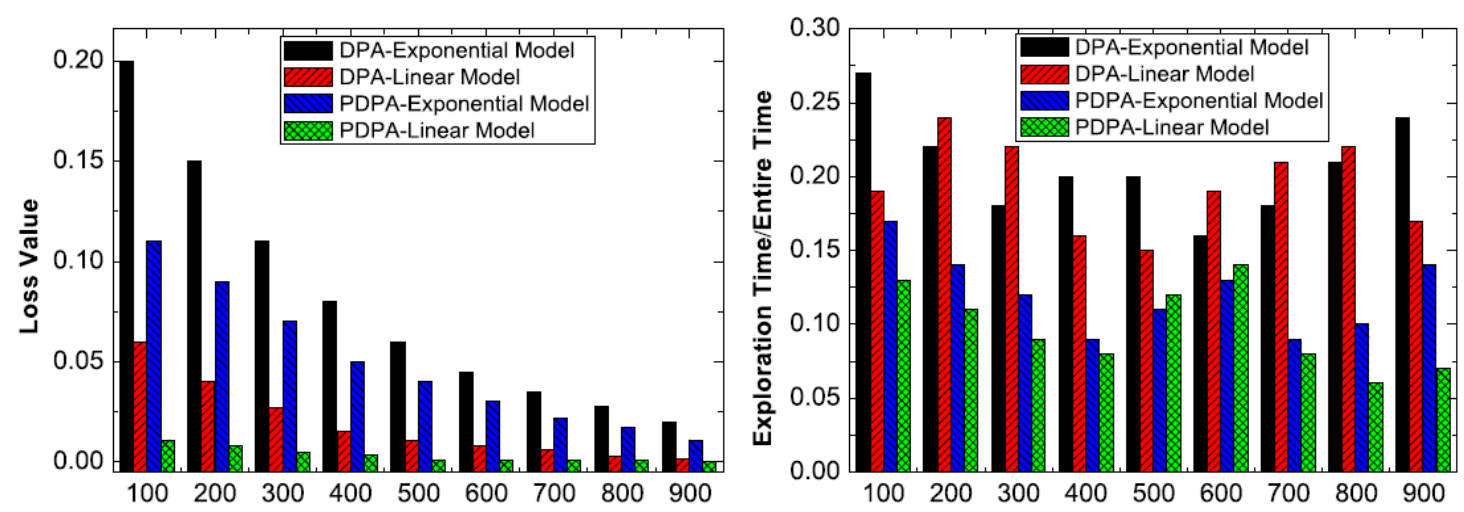

Figure 7. The Numerical Simulation Result of the Proposed System

\section{Conclusion}

In this paper, we research on the modern Internet of things system structure optimization methodology based on the information theory and the communication signal transmission model. Although the Internet of things so far have no a unified standard definition, but from the point of view of its essence, it is the modern information technology development to a certain stage after the polymerized application and a technology promotion. The Internet of things will be all kinds of integration technology of polymerization and application, such as perception technology, modern network technology, the artificial intelligence and automation technology, make the person dialogue with specific content, creating a dynamic world. We optimize the traditional structures of the systems from following layers. (1) The application layer, application layer mainly includes application support platform and the application service layer. The application support platform layer used to support the cross-industry, across application, information synergy between across systems, sharing and communication function. (2) Public technology layer: the technology does not belong to a particular aspect of the Internet of things technology, but with the Internet of things technology architecture of the three layers have relations, it includes the identification and resolution, security technology, network management and the service quality management. (3) The perception layer: the data acquisition and perception is mainly used for the physical events that took place in the physical world and data, including all kinds of physical quantities, identification, audio and video data. (4) Network layer: it is to be able to perceive the information accessibility, high reliability and high security which need the sensor networks and the general integration of the mobile communication technology. In the experimental part, we test and verify effectiveness and feasibility of the architecture. In the recent future, we plan to focus on perception layer of the IOT system to modify the tags and sensors adopted by the system.

\section{Acknowledgement}

This research is supported by the 2014 major teaching reform project in Anhui province (2014zdjy177); the education revitalization plan in 2014 in Anhui province professional leader funded project. 


\section{References}

[1] L. Atzori, A. Iera and Giacomo Morabito, "Siot: Giving a social structure to the internet of things", Communications Letters, IEEE, vol. 15, no. 11, (2011), pp. 1193-1195.

[2] J. Gubbi, R. Buyya, S. Marusic and M. Palaniswami, "Internet of Things (IoT): A vision, architectural elements, and future directions", Future Generation Computer Systems, vol. 29, no. 7, (2013), pp. 16451660 .

[3] C. Kuang and W. Li, "Application of Formalization Method in Construction ZigBee Technology and RFID System in Internet of Things", In 2015 International Conference on Mechatronics, Electronic, Industrial and Control Engineering (MEIC-15). Atlantis Press, (2015).

[4] S. Li, T. Xia, C. Z. Tang, X. Huang and Min Zhou, "Study on Bridge Structural Safety Monitoring Network Platform Based on Fiber Optic Sensing and Internet of Things", In Applied Mechanics and Materials, vol. 602, (2014), pp. 1980-1983.

[5] X. Wang, "The architecture design of the wearable health monitoring system based on internet of things technology", International Journal of Grid and Utility Computing 6, no. 3-4, (2015), pp. 207-212.

[6] R. Khan, S. Khan, R. Zaheer and S. Khan, "Future Internet: the Internet of Things architecture, possible applications and key challenges", In Frontiers of Information Technology (FIT), 2012 10th International Conference on, IEEE, (2012), pp. 257-260.

[7] Q. Hao, F. Zhang, Z. Liu and Lele Qin, "Design of Chemical Industrial Park Integrated Information Management Platform Based on Cloud Computing and IOT (The Internet of Things) Technologies", International Journal of Smart Home 9, no. 4, (2015).

[8] S. Zhongyan and Ding Lei, "Strategy Research of China's Internet of Things Industry Under the Perspective of the Profit Pool", Reformation \& Strategy, vol. 5, (2013).

[9] Z. Sheng, S. Yang, Y. Yu, A. Vasilakos, J. Mccann and Kin Leung, "A survey on the item protocol suite for the internet of things: Standards, challenges, and opportunities", Wireless Communications, IEEE 20, no. 6 (2013), pp. 91-98.

[10] N. Lin and W. Shi, "The research on Internet of things application architecture based on web." In Advanced Research and Technology in Industry Applications (WARTIA), 2014 IEEE Workshop on, (2014), pp. 184-187.

[11] J. Sun, "Design and implementation of physical evidence management system based on the Internet of Things intelligent perception technology", In Computer Science and Automation Engineering (CSAE), 2012 IEEE International Conference on, vol. 3, (2012), pp. 604-608.

[12] J. Kang, S. Yin, and W. Meng, "An Intelligent Storage Management System Based on Cloud Computing and Internet of Things", In Proceedings of International Conference on Computer Science and Information Technology, (2014), pp. 499-505.

[13] Z. Bi, L. D. Xu and C.Wang, "Internet of Things for enterprise systems of modern manufacturing", Industrial Informatics, IEEE Transactions on 10, no. 2 (2014), pp. 1537-1546.

[14] Y. Guo, H. Liu and Y Chai, "The embedding convergence of smart cities and tourism internet of things in China: An advance perspective", Advances in Hospitality and Tourism Research, vol. 2, no. 1, (2014), pp. 54-69.

[15] X. Y. Ai, D. S. Xu, F. E. N. G. Zhang and J. G. Dong, "Agriculture Intelligent Control System Algorithm for Wireless Sensor Networks Based on Internet of Things", Sensors \& Transducers (17265479), vol. 158, no. 11, (2013).

[16] Y. Chen, J. Zhou and M. Guo, "A context-aware search system for Internet of Things based on hierarchical context model”, Telecommunication Systems, (2015), pp. 1-15.

[17] C. Hochleitner, C. Graf, D. Unger and M. Tscheligi, "Making Devices Trustworthy: Security and Trust Feedback in the Internet of Things", In Fourth International Workshop on Security and Privacy in Spontaneous Interaction and Mobile Phone Use (IWSSI/SPMU), Newcastle, UK, (2012). 
International Journal of Future Generation Communication and Networking Vol. 9, No.9, (2016) 